\title{
Real-world evidence of NEPA, netupitant-palonosetron, in chemotherapy-induced nausea and vomiting prevention: effects on quality of life
}

\author{
Meinolf Karthaus*,1,2, Gülten Oskay-Özcelik ${ }^{3}$, Pia Wülfing ${ }^{4}$, Carsten Hielscher ${ }^{5}$, Dagmar \\ Guth $^{6}$, Mark-Oliver Zahn ${ }^{7}$, Elisa Flahaut ${ }^{8}$ \& Jörg Schilling ${ }^{9}$ \\ ${ }^{1}$ Department of Hematology, Oncology \& Palliative Care, Klinikum Neuperlach, Munich, Germany \\ ${ }^{2}$ Department of Hematology, Oncology \& Palliative Care, Klinikum Harlaching, Munich, Germany \\ ${ }^{3}$ Gynecological Oncology Practice, Berlin, Germany \\ ${ }^{4}$ Mammazentrum Hamburg am Krankenhaus Jerusalem, Hamburg, Germany \\ ${ }^{5}$ Gynecological Oncology Center, Stralsund, Germany \\ ${ }^{6}$ Gynecological Oncology Practice, Plauen, Germany \\ ${ }^{7}$ Oncology Center Harz, Goslar, Germany \\ ${ }^{8}$ Department of Medical Affairs, RIEMSER Pharma GmbH, Berlin, Germany \\ ${ }^{9}$ Gynecological Oncology Practice, Berlin, Germany \\ *Author for correspondence: meinolf.karthaus@klinikum-muenchen.de
}

\begin{abstract}
Aim: To determine quality of life, effectiveness and safety of oral netupitant-palonosetron (NEPA)-based antiemetic prophylaxis in the real-world setting. Materials \& methods: Prospective, noninterventional study in adults receiving highly or moderately emetogenic chemotherapy and NEPA for three cycles. NEPA was administered per summary of product characteristics. Results: A total of 2429 patients enrolled, 2173 were evaluable. 'No impact on daily life' due to vomiting was reported by $84 \% / 82 \%$ of patients in the highly emetogenic chemotherapy/moderately emetogenic chemotherapy groups in cycle 1, with rates of $54 \% / 59 \%$ for nausea. Overall, complete response rate was $89 \% / 87 \% / 83 \%$ in the acute/delayed/overall phases. NEPA was well tolerated. Conclusion: NEPA had beneficial effects on the quality of life of a heterogeneous group of cancer patients and was safe and effective in the real-world setting.
\end{abstract}

First draft submitted: 3 March 2020; Accepted for publication: 27 March 2020; Published online: 16 April 2020

Keywords: CINV • effectiveness $\bullet$ FLIE $\bullet$ highly emetogenic chemotherapy $\bullet$ moderately emetogenic chemotherapy - NEPA • quality of life $\bullet$ real world

Chemotherapy-induced nausea and vomiting (CINV) is a common and distressing side effect that has a detrimental impact on quality of life [1-5] and may contribute to negatively influence the overall survival of patients [6]. Recent advances in antiemetic research have led to important improvements in the control of vomiting, although nausea remains a clinical challenge and represents an unmet medical need [7]. Clear benefits have been observed in patients who have received prophylactic antiemetic treatment in accordance with the evidence-based guidelines [8-11] published by key international oncology associations, including the Multinational Association of Supportive Care in Cancer (MASCC) and the European Society for Medical Oncology (ESMO) [12,13], the American Society of Clinical Oncology (ASCO) [14] and the National Comprehensive Cancer Network (NCCN) [15]. These recommendations all base their guidance on the potential of a chemotherapeutic agent to cause emesis within the first $24 \mathrm{~h}$ after administration in the absence of antiemetic prophylaxis [16-18].

In general, for patients receiving highly emetogenic chemotherapy (HEC), which also includes those on anthracycline-cyclophosphamide (AC)-based regimens, a triple combination of a neurokinin-1 receptor antagonist $\left(\mathrm{NK}_{1} \mathrm{RA}\right)$, a 5-hydroxytryptamine-3 (5- $\left.\mathrm{HT}_{3}\right) \mathrm{RA}$ and dexamethasone is recommended in guidelines from the NCCN [15] and MASCC/ESMO [12,13]. A four-drug regimen consisting of olanzapine added to the $\mathrm{NK}_{1}$ RA-5- $\mathrm{HT}_{3} \mathrm{RA}-\mathrm{dexamethasone}$ combination is recommended for these patients in the ASCO guidelines [14]. The

Future Medicine 
addition of olanzapine to the triplet combination has also been advised, particularly in patients who experience breakthrough nausea and vomiting [14,15] or in those for whom nausea is a concern [13]. The $\mathrm{NK}_{1} \mathrm{RA}-5-\mathrm{HT}_{3}$ RA-dexamethasone triplet combination is recommended for those patients receiving carboplatin-based regimens at all carboplatin doses [12,13], or only at doses of area under the concentration-time curve $\geq 4 \mathrm{mg} / \mathrm{ml} / \mathrm{min}[14,15]$. For patients who receive moderately emetogenic chemotherapy (MEC), a combination of $5-\mathrm{HT}_{3} \mathrm{RA}$ plus dexamethasone is recommended. It is also proposed that an $\mathrm{NK}_{1} \mathrm{RA}$ is included in patients who have additional risk factors or for whom previous 5- $\mathrm{HT}_{3}$ RA plus dexamethasone treatment has failed [15].

Despite the existence of the various antiemetic guidelines, several prospective and retrospective studies have demonstrated that adherence to them in clinical practice is largely suboptimal, and this can result in uncontrolled CINV [8-10,19,20]. One recent analysis based on real-world data from 2018 of antiemetic prescription patterns among physicians in Europe found that adherence to clinical guidelines is often unsatisfactory in each of the chemotherapy settings. $\mathrm{NK}_{1}$ RA use was reported in just 45,42 and $19 \%$ of patients receiving cisplatin-, AC- and carboplatin-based chemotherapy treatments, respectively; $\mathrm{NK}_{1} \mathrm{RA}$ use was highest in Germany - in 61, 69 and $47 \%$ of patients treated with cisplatin-, AC- and carboplatin-based chemotherapy regimens, respectively [20,21]. A study by Dranitsaris et al. analyzed data from five studies undertaken between 2008 and 2015, and found that in the HEC setting, only $12 \%$ of patients received triplet antiemetic prophylaxis [22]. Furthermore, oncologists in Europe, as well as oncology nurses in the US and Europe, have all reported low adherence to antiemetic guidelines in the HEC and MEC settings [23,24].

The complexity of the $\mathrm{NK}_{1}$ RA-based administration schedules may be a reason for the low adherence to antiemetic guidelines, and a likely cause of the guidelines-inconsistent antiemetic prescription patterns by physicians. It also increases the potential for noncompliance with antiemetic treatments by patients [19,25]. In a European survey that included 386 cancer patients, only $38 \%$ of participants reported taking their antiemetic drugs at home as prescribed by their physician or oncology nurse [25]. Similarly, a survey among European oncologists revealed that approximately only a third of patients comply with antiemetic treatments during home administration, and that patients commonly make administration mistakes when taking antiemetics at home [19]. Currently, there are three oral $\mathrm{NK}_{1}$ RAs approved and recommended by antiemetic guidelines: aprepitant, rolapitant and netupitant (in fixed combination with palonosetron, netupitant-palonosetron [NEPA]). These agents differ in the complexity of their administration schedule, which is largely determined by their distinct pharmacologic properties.

NEPA is the only available fixed-combination antiemetic. It is composed of an $\mathrm{NK}_{1} \mathrm{RA}$, netupitant (300 mg) and a $5-\mathrm{HT}_{3} \mathrm{RA}$, palonosetron $(0.50 \mathrm{mg})$; thus, it acts by blocking two main emetic pathways in a single dose [26]. Oral NEPA single dose and dexamethasone $(12 \mathrm{mg}$ ) are administered on day 1 (MEC and HEC), and in the non-AC HEC setting, single-dose dexamethasone ( $8 \mathrm{mg}$ ) is also given on days 2-4 [27-30]. Among the $\mathrm{NK}_{1} \mathrm{RAs}_{\text {, }}$ NEPA-based regimens are the simplest, as they require the fewest doses [27-34]. Therefore, NEPA provides a more straightforward and convenient antiemetic treatment schedule that may facilitate the uptake and prescription of guidelines-recommended prophylaxis and minimize the risk for mistakes by patients. The efficacy of oral NEPA and dexamethasone for the prevention of CINV associated with single and multiple cycles of HEC (AC and non-AC) and MEC has been well established in a number of Phase II and III randomized controlled trials [27-30,35]. In these studies, the tolerability and safety profile of oral NEPA was also determined in over 1500 NEPA-treated patients [27-29]. Oral NEPA was approved by the US FDA in 2014 and the European Medicines Agency (EMA) in 2015. An IV NEPA (fosnetupitant-palonosetron) formulation has also been developed [36,37] and was approved by the FDA [38] in 2018 and the EMA in 2019 [39]; it is recommended by international guidelines for the prevention of CINV associated with HEC and MEC [13,15].

While information gathered from randomized controlled trials is both invaluable and essential for securing drug approval by regulatory agencies, real-world data showing the impact of antiemetics on cancer patients outside of clinical trials are scarce. Noninterventional studies have great value for the clinical community, as they can better inform about the effectiveness and safety of the antiemetics in the real world. Because they enroll patients with diverse baseline characteristics and clinical settings, and as they are conducted in the absence of external supervision (which ensures correct and timely drug administration), evidence of treatment compliance by patients can be collected. Improving patient quality of life during chemotherapy treatment is the goal of cancer supportive care, but it is always considered a secondary end point in clinical trial design [40]. Patient self-reporting of chemotherapy-related adverse symptoms, including nausea and vomiting, followed by timely clinical management has been associated with improved quality of life, more-successful completion rates of chemotherapy and prolonged survival [6,41]. 
We have conducted a large noninterventional study to evaluate quality of life in patients receiving NEPA-based antiemetic prophylaxis for CINV in a real-world setting through the prospective collection of patient-reported nausea and vomiting symptoms. Treatment effectiveness and safety were also analyzed.

\section{Materials \& methods}

\section{Study design}

This prospective, noninterventional study was conducted at 162 sites throughout Germany from September 2015 to March 2018. The aim of the study was to determine the quality of life of adult cancer patients receiving NEPA for the prevention of nausea and vomiting associated with HEC or MEC in daily clinical practice. Secondary objectives included evaluating effectiveness, as determined by measuring the rate of complete response (CR) and the nature of rescue medication, as well as safety outcomes. Patients provided signed informed consent before enrollment. The study was conducted in accordance with the German Medicines Act (AMG), and the Supreme Federal Authority, the National Association of Statutory Health Insurance Physicians (KBV), the Central Federal Association of Statutory Health Insurance Funds (GKV-Spitzenverband) and the Association of Private Health Insurers (PKV) were notified by the clinical research organization OnkoDataMed GmbH, Germany.

\section{Patients \& procedures}

Recruitment took place between September 2015 and September 2017. Eligible patients were: male and female adults; naive and nonnaive to chemotherapy; with solid or hematologic cancers; receiving anticancer treatment in an outpatient or inpatient setting; and receiving 1- or 2-day HEC or MEC regimens and NEPA-based antiemetic prophylaxis, which needed to be scheduled prior to enrollment. Among patients receiving HEC, only those treated with AC- (considered MEC at the time of study start) or cisplatin-based HEC regimens were included in the analysis population, as per summary of product characteristics ( $\mathrm{SmPC}$ ); patients treated with other HEC regimens were excluded from the analysis. Patients planned for treatment with a MEC regimen could be enrolled at the investigator's discretion on the basis of clinical criteria. Patients with pre-existing anticipatory nausea and vomiting were excluded, as well as those with nonchemotherapy-related nausea and vomiting. Additional exclusion criteria were: receiving radiotherapy alone; presence of brain tumors or symptomatic metastases in the brain; scheduled to receive chemotherapy treatments that are longer than 2 days per cycle; pregnant women; known hypersensitivity to active substances, excipients or other ingredients present in NEPA.

The study did not influence any medical decisions or procedures by the treating physician and had no impact on the patients' treatment regimen. NEPA was administered as per the SmPC. One oral dose was administered approximately $1 \mathrm{~h}$ before the start of each chemotherapy cycle with dexamethasone $(12 \mathrm{mg})$ on day 1 . For non-AC HEC, dexamethasone ( $8 \mathrm{mg}$ ) once daily was also administered on days $2-4$.

\section{Assessments}

The primary outcome was quality of life as measured via the Functional Living Index-Emesis (FLIE) questionnaire [42], which was completed by patients on days 1 through 5 for 1-day chemotherapies, and days 1 through 6 for 2-day chemotherapies. In brief, patients were asked to complete 18 questions related to the impact on daily life due to nausea and vomiting (nine questions for each domain) using a visual analog scale with points 1 ('not at all') to 7 ('a great deal'). Effectiveness parameters were recorded in patient diaries and included information regarding the frequency and severity (graded as severe, moderate, mild or none) of nausea and vomiting before and after chemotherapy, as well as the use of rescue medication and its nature. Physicians' and patients' perceptions of the effectiveness of NEPA prophylaxis was measured by a four-point scale (very good, good, satisfactory or bad). For safety assessments, all adverse events (AEs) regardless of their relationship to NEPA that occurred on days 1 through 21 of each chemotherapy cycle were documented in electronic case report forms. The relationship to the study drug was determined by the investigator. Quality of life, effectiveness and safety data for individual patients were collected in electronic case report forms during three chemotherapy cycles. For patients who received NEPA for fewer than three cycles, the reason for treatment discontinuation was recorded.

\section{Statistical analysis}

The planned enrollment was 2500 patients, recruited at approximately 200 sites across Germany, with each site recruiting a minimum of ten and a maximum of 20 patients. No formal sample size calculation was performed. The target patient enrollment was considered to be adequate for the exploratory purposes of the study regarding 


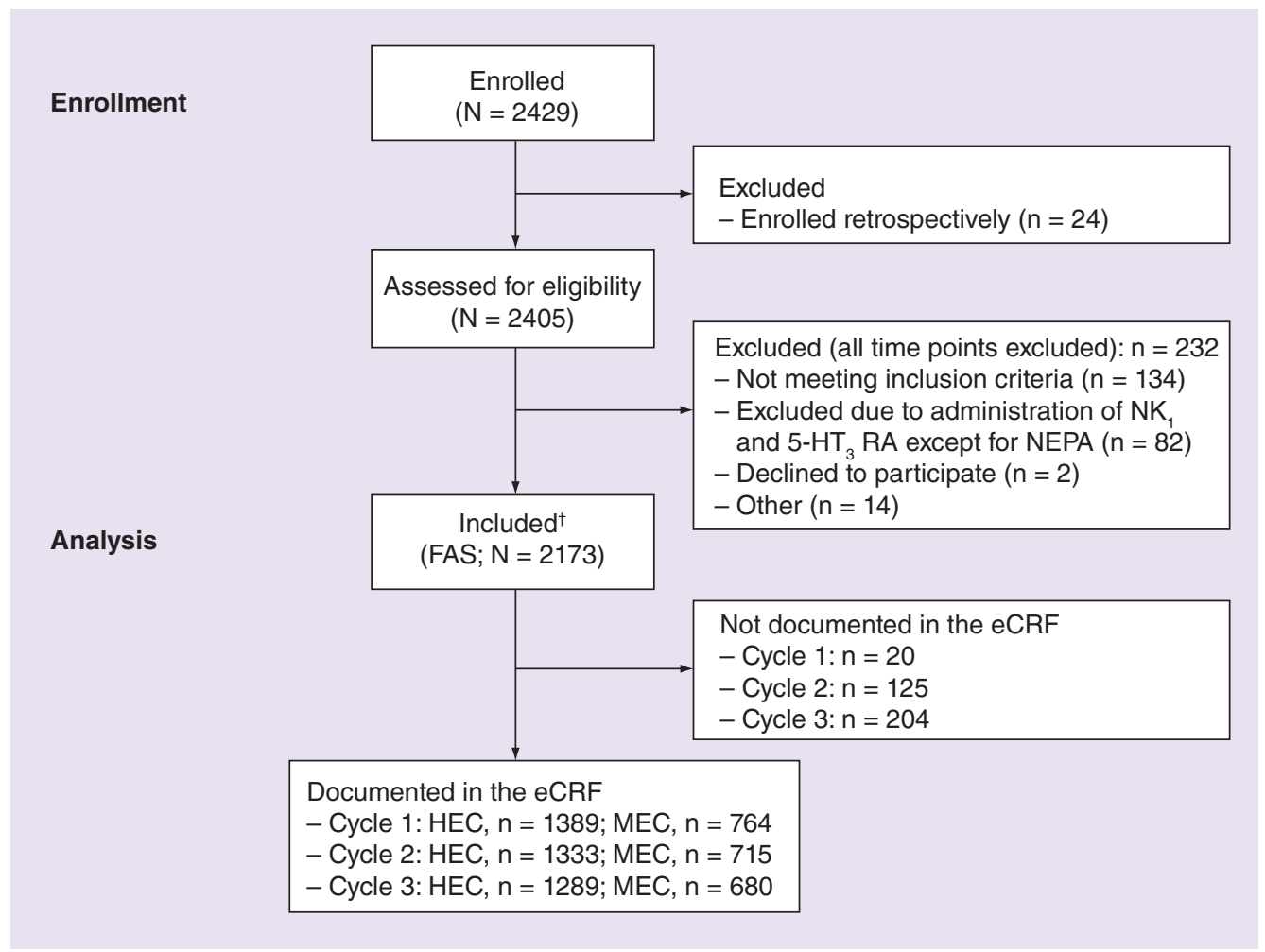

Figure 1. Patient disposition.

${ }^{\dagger}$ For 187 patients, study discontinuation at any of the three cycles was documented.

5- $\mathrm{HT}_{3}$ : 5-hydroxytryptamine-3; eCRF: Electronic case report form; HEC: Highly emetogenic chemotherapy; MEC:

Moderately emetogenic chemotherapy; NEPA: Netupitant-palonosetron; NK ${ }_{1}$ : Neurokinin-1; RA: Receptor antagonist.

the quality of life of patients receiving NEPA. The number of sites and recruitment limits per site were considered to be appropriate to ensure that the data were representative for Germany. Analysis of demographic, quality of life, effectiveness and safety data was performed descriptively, and statistical significance was not formally evaluated. Categoric data were presented with absolute and relative frequencies. Patient stratification according to the emetogenicity of chemotherapy (HEC vs MEC) was performed for quality of life analyses.

The FLIE questionnaires were analyzed according to the FLIE scoring and administration manual v12; higher scores correlated to better performance on daily life activities due to the lower occurrence of nausea and vomiting. 'No impact on daily life (NIDL) activities' for individual nausea and vomiting domains was defined as a FLIE score higher than 53.8; NIDL for the combined domains of nausea and vomiting was defined as a FLIE score higher than 108. NIDL analysis was performed separately for the HEC and MEC groups; patients were allocated to either group on the basis of the emetogenicity of the chemotherapeutic regimen received in cycle 1 . For effectiveness outcomes, CR was defined as no emesis and no rescue medication, and 'no significant nausea (NSN)' was defined as no or mild nausea.

Regarding statistical analysis, missing values in the FLIE questionnaire were handled as per the instruction manual. Missing values for the rest of the parameters were not replaced, and the number of patients without missing values is provided.

\section{Results}

Patients

A total of 2429 patients were enrolled at 162 study centers across Germany, 2405 of whom were assessed for eligibility; of these, 2173 were included in the final analysis and constitute the full analysis set population. Patient disposition is represented in Figure 1. Patient demographics and baseline clinical characteristics are summarized in Table 1 . The median age was 58 years (range, 25-89 years), the majority of patients were female (85\%), and the most common tumor type was breast cancer $(66 \%)$. Approximately $25 \%$ of patients presented with metastatic 
Table 1. Patient demographics and baseline characteristics - full analysis set population.

Parameter

Overall population $(n=2173)$

\section{Female}

$1853(85.3)$

Male

320 (14.7)

Age, years

Mean (SD)

57.7 (12.12)

Median (range)

58.0 (25-89)

ECOG PS, n (\%)

0

1

2

3

Tumor type, n (\%)

Breast

1334 (61.4)

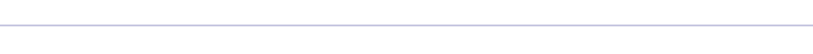

$722(33.2)$

$109(5.0)$

$8(0.4)$

Ovarian

$1430(65.8)$

Colorectal

$157(7.2)$

Bronchial

$132(6.1)$

Cervix

67 (3.1)

Stomach

53 (2.4)

Stom

50 (2.3)

Lung

37 (1.7)

Non-Hodgkin lymphoma

34 (1.6)

Other

$213(9.8)$

Extent of tumor, n (\%)

Primary

1456 (67.0)

Metastasis

542 (24.9)

Missing

175 (8.1)

Therapy type $^{\dagger}, \mathrm{n}$ (\%)

Adjuvant

994 (45.7)

Neoadjuvant

714 (32.9)

Palliative

Number of patients per cycle, cycle $1 / 2 / 3^{\ddagger}$

445 (20.5)

Emetic risk of chemotherapy ${ }^{\ddagger}$, no. of patients in cycle $1 / 2 / 3(\%)$

$2153 / 2048 / 1969$

HEC (total)

$1389 / 1333 / 1289$

(64.5/65.1/65.5)

- HEC-cisplatin

$164 / 146 / 136$

(7.6/7.1/6.9)

- HEC-AC

$1225 / 1187 / 1153$

(56.9/58.0/58.6)

MEC (total)

$764 / 715 / 680$

(35.5/34.9/34.5)

- MEC-carboplatin

$406 / 382 / 368$

(18.9/18.7/18.7)

- MEC-oxaliplatin

$164 / 150 / 137$

(7.6/7.3/7.0)

- MEC-other

$194 / 183 / 175$

(9.0/8.9/8.9)

$\dagger$ Patients receiving multiple therapies at cycles 1,2 , and 3 could be assigned to multiple categories.

$\ddagger$ Patients not documented in the eCRF were excluded.

$\S$ Nausea and vomiting directly before the start of chemotherapy.

INausea 1 day before the start of chemotherapy.

AC: Anthracycline and cyclophosphamide; CINV: Chemotherapy-induced nausea and vomiting; ECOG PS: Eastern Cooperative Oncology Group performance status; eCRF: Electronic case report form; HEC: Highly emetogenic chemotherapy; MEC: Moderately emetogenic chemotherapy; SD: Standard deviation. 
Table 1. Patient demographics and baseline characteristics - full analysis set population (cont.).

\begin{tabular}{|c|c|}
\hline Parameter & Overall population $(n=2173)$ \\
\hline \multicolumn{2}{|l|}{ Chemotherapy schedule $\ddagger$, no. of patients in cycle $1 / 2 / 3(\%)$} \\
\hline 1-day & $\begin{array}{l}1958 / 1871 / 1802 \\
(90.9 / 91.4 / 91.5)\end{array}$ \\
\hline 2-day & $\begin{array}{l}195 / 177 / 167 \\
(9.1 / 8.6 / 8.5)\end{array}$ \\
\hline $\begin{array}{l}\text { Anticipatory CINV } \ddagger, \S \text {, percentage of patients in cycle } 1 / 2 / 3 \\
\text { Nausea } \\
\text { Vomiting }\end{array}$ & $\begin{array}{l}8.8 / 8.8 / 10.7 \\
2.7 / 2.5 / 1.9\end{array}$ \\
\hline Previous nausea ${ }^{\ddagger} \mathbb{I}$, percentage of patients in cycle $1 / 2 / 3$ & $10.8 / 9.3 / 10.2$ \\
\hline \multicolumn{2}{|c|}{$\begin{array}{l}\text { †Patients receiving multiple therapies at cycles } 1,2 \text {, and } 3 \text { could be assigned to multiple categories. } \\
\text { ¥Patients not documented in the eCRF were excluded. } \\
\text { §Nausea and vomiting directly before the start of chemotherapy. } \\
\text { INausea } 1 \text { day before the start of chemotherapy. } \\
\text { AC: Anthracycline and cyclophosphamide; CINV: Chemotherapy-induced nausea and vomiting; ECOG PS: Eastern Cooperative Oncology Group performance status; eCRF: Electronic case } \\
\text { report form; HEC: Highly emetogenic chemotherapy; MEC: Moderately emetogenic chemotherapy; SD: Standard deviation. }\end{array}$} \\
\hline
\end{tabular}

disease at study entry, and $21 \%$ were treated in the palliative setting. A total of 1389 patients received HEC and 764 MEC in cycle 1 (Figure 1). AC-HEC and carboplatin-based MEC were the most common regimens in each cycle. The majority of patients (91\%) were scheduled to receive 1-day chemotherapy regimens at study entry. Between 9 and $11 \%$ of patients in cycles 1 through 3 experienced nausea at the start of chemotherapy or on the day before. A minority of patients received concomitant immunotherapy, with the most common (in $\geq 1 \%$ of patients in cycles $1-3)$ agents being bevacizumab (3-4\%), trastuzumab (3\% for all cycles), pertuzumab ( $2 \%$ for all cycles) and rituximab $(1-2 \%)$.

\section{Eastern Cooperative Oncology Group}

Regarding performance status, most patients had Eastern Cooperative Oncology Group (ECOG) 0 (61\%) or 1 (33\%) at study entry (Supplementary Figure 1). As expected, during chemotherapy treatment, the percentage of patients with ECOG 0 gradually decreased to $48 \%$, and the percentage of patients with ECOG 1 gradually increased to similar levels in cycle $3(46 \%)$. A lower proportion of patients had ECOG $2(5 \%)$ or $3(0.4 \%)$ at study entry, which remained nearly unchanged across cycles 1 through 3.

Quality of life

Evaluable FLIE questionnaires were collected from $1886(88 \%)$ patients in cycle 1, $1795(88 \%)$ patients in cycle 2 and $1698(86 \%)$ patients in cycle 3. NIDL due to vomiting during cycle 1 was reported by $84 \%$ of patients in the HEC group and 82\% in the MEC group. These frequencies were maintained in cycles 2 and 3 (Figure 2A). Meanwhile, NIDL due to nausea increased from 54\% in cycle 1 to $58 \%$ in cycle 3 for patients receiving HEC, and from $59 \%$ in cycle 1 to $66 \%$ in cycle 3 for patients in the MEC group (Figure 2B). The rates reported for the combined domain of NIDL due to nausea and vomiting were consistent across cycle $1(64 \%)$ and in cycles 2 and 3 (66\% in each cycle) for patients in the HEC group, while the rates increased from $67 \%$ in cycle 1 to 73 and $74 \%$ in cycles 2 and 3, respectively, for patients receiving MEC (Figure 2C).

\section{Effectiveness}

The CR rate (no emesis and no use of rescue medication) was $89 \%$ in the acute phase $(0-24 \mathrm{~h}), 87 \%$ in the delayed phase $(25-120 \mathrm{~h})$ and $83 \%$ in the overall period $(0-120 \mathrm{~h})$ in cycle 1 (Figure 3$)$. The effectiveness outcomes after NEPA prophylaxis are summarized in Table 2 . In cycle 1 , the percentage of patients without emesis was high in both the acute and delayed phases ( $96 \%$ in each), while the rates of NSN and no nausea decreased slightly between the acute and delayed phases (NSN: 79\%/75\%; no nausea: 56\%/44\%). In the overall period, the majority of patients reported no emesis (94\%), and the rates of NSN and no nausea were 67 and 38\%, respectively. Rescue medication was required for $7.5,10$ and $13 \%$ of patients in the acute, delayed and overall phases, respectively. Metoclopramide was the most frequently used agent, followed by dimenhydrinate and ondansetron. The rates of all the effectiveness parameters that were analyzed were maintained in cycles 2 and 3 . As reported in patients' diaries, the rates and severity of nausea decreased gradually from day 1 through 5 in cycle 1 . Similar results were observed for cycles 2 and 3 (Figure 4). The majority of physicians $(\geq 89 \%)$ and patients $(\geq 86 \%)$ rated the effectiveness of 

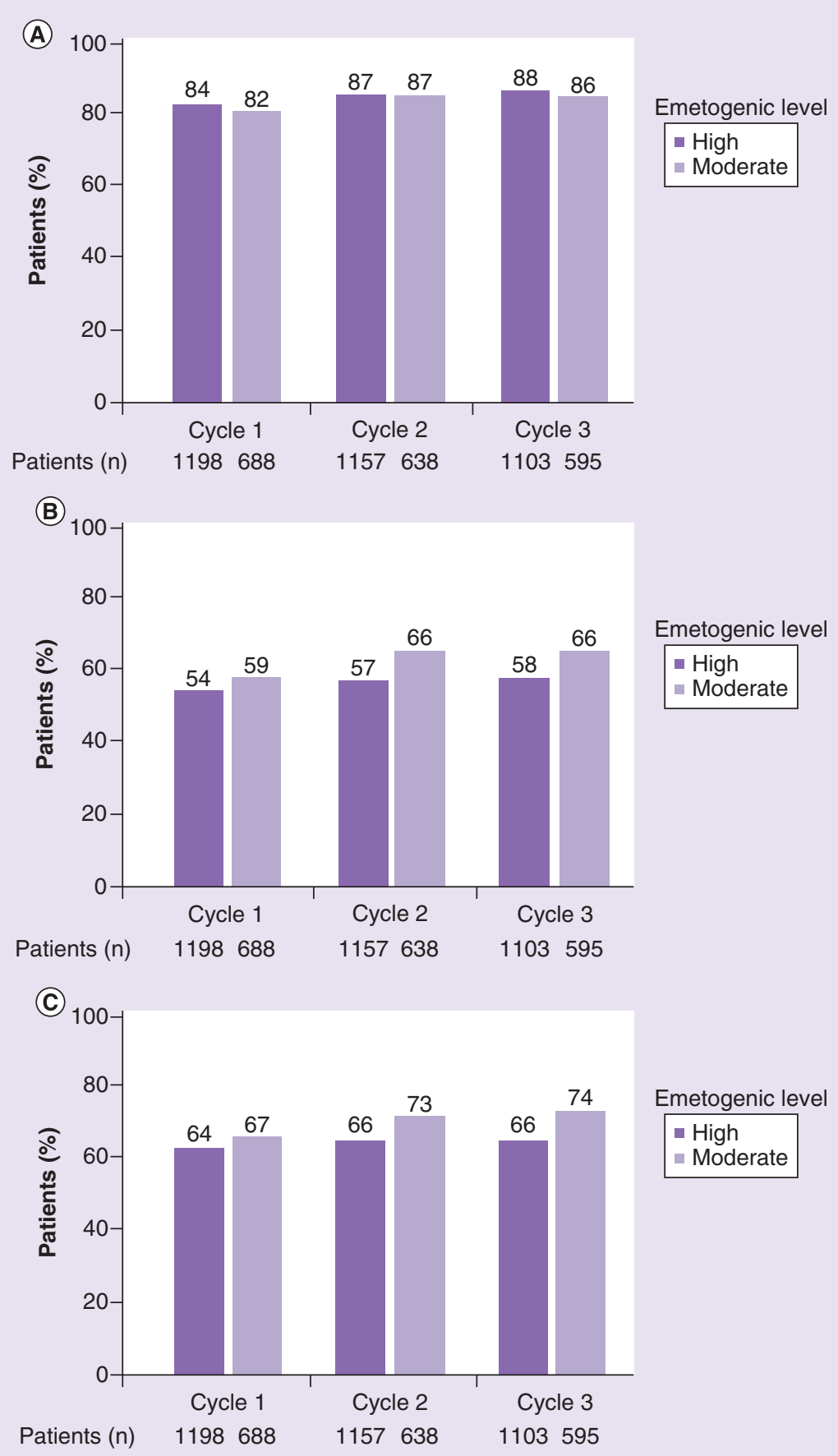

Figure 2. Relative frequencies of HEC and MEC patients without any influence on their daily life due to vomiting. (A) Nausea; (B) nausea and vomiting; (C) by cycle and emetogenic levels - FAS population.

FAS: Full analysis set; FLIE: Functional living index-emesis; HEC: Highly emetogenic chemotherapy; MEC: Moderately emetogenic chemotherapy.

NEPA prophylaxis as 'very good' or 'good' during all three chemotherapy cycles (Figure 5), and the perception of antiemetic control by NEPA was seen to be similar between physicians and patients at each cycle. 


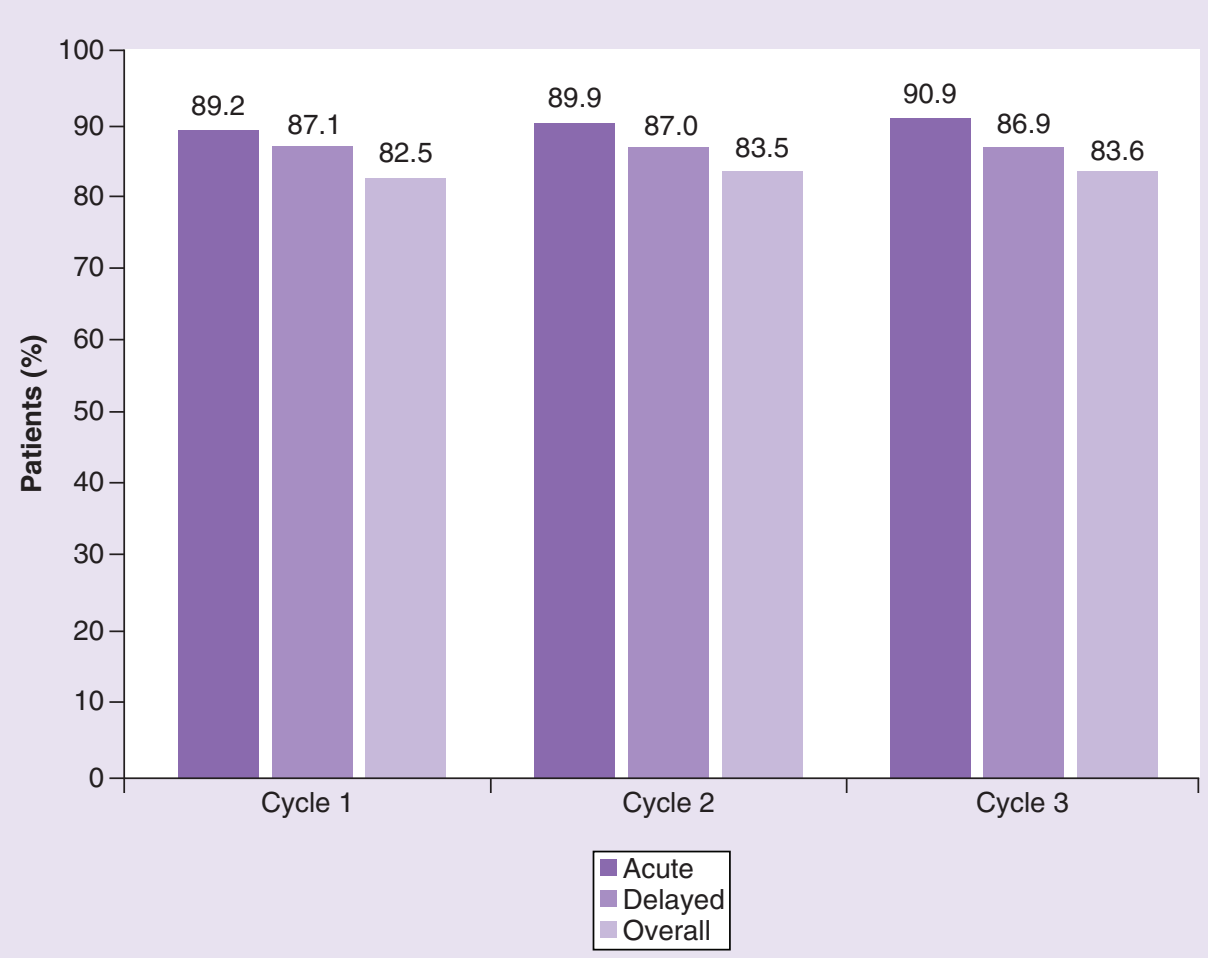

Figure 3. Complete response rate in the acute $(0-24 \mathrm{~h})$, delayed $(25-120 \mathrm{~h})$ and overall $(0-120 \mathrm{~h})$ period - FAS population.

FAS: Full analysis set.

\begin{tabular}{|c|c|c|c|}
\hline Parameter, patients (\%) & $\begin{array}{l}\text { Cycle } 1 \\
(n=2153)\end{array}$ & $\begin{array}{l}\text { Cycle } 2 \\
(n=2048)\end{array}$ & $\begin{array}{l}\text { Cycle } 3 \\
(n=1969)\end{array}$ \\
\hline \multicolumn{4}{|l|}{ No emesis } \\
\hline Acute & 95.7 & 96.2 & 96.9 \\
\hline Delayed & 96.1 & 95.8 & 96.4 \\
\hline Overall & 93.5 & 93.6 & 94.7 \\
\hline \multicolumn{4}{|l|}{ No significant nausea } \\
\hline Acute & 78.8 & 81.8 & 83.1 \\
\hline Delayed & 74.8 & 75.1 & 74.5 \\
\hline Overall & 66.9 & 68.7 & 69.2 \\
\hline \multicolumn{4}{|l|}{ No nausea } \\
\hline Acute & 55.7 & 58.6 & 59.8 \\
\hline Delayed & 44.3 & 47.2 & 47.2 \\
\hline Overall & 38.4 & 41.6 & 43.1 \\
\hline \multicolumn{4}{|l|}{ No rescue medication } \\
\hline Acute & 92.5 & 93.1 & 94.0 \\
\hline Delayed & 90.0 & 90.0 & 91.2 \\
\hline Overall & 87.0 & 87.5 & 88.2 \\
\hline
\end{tabular}

\section{Safety}

In total, $650(30 \%)$ patients reported treatment-emergent AEs during the study period; of these, 158 (7\%) experienced NEPA treatment-related AEs (TRAEs). A summary of the overall safety data is shown in Table 3 . The majority of treatment-emergent AEs (86\%) were of mild or moderate intensity. Of the $153(7 \%)$ patients who 


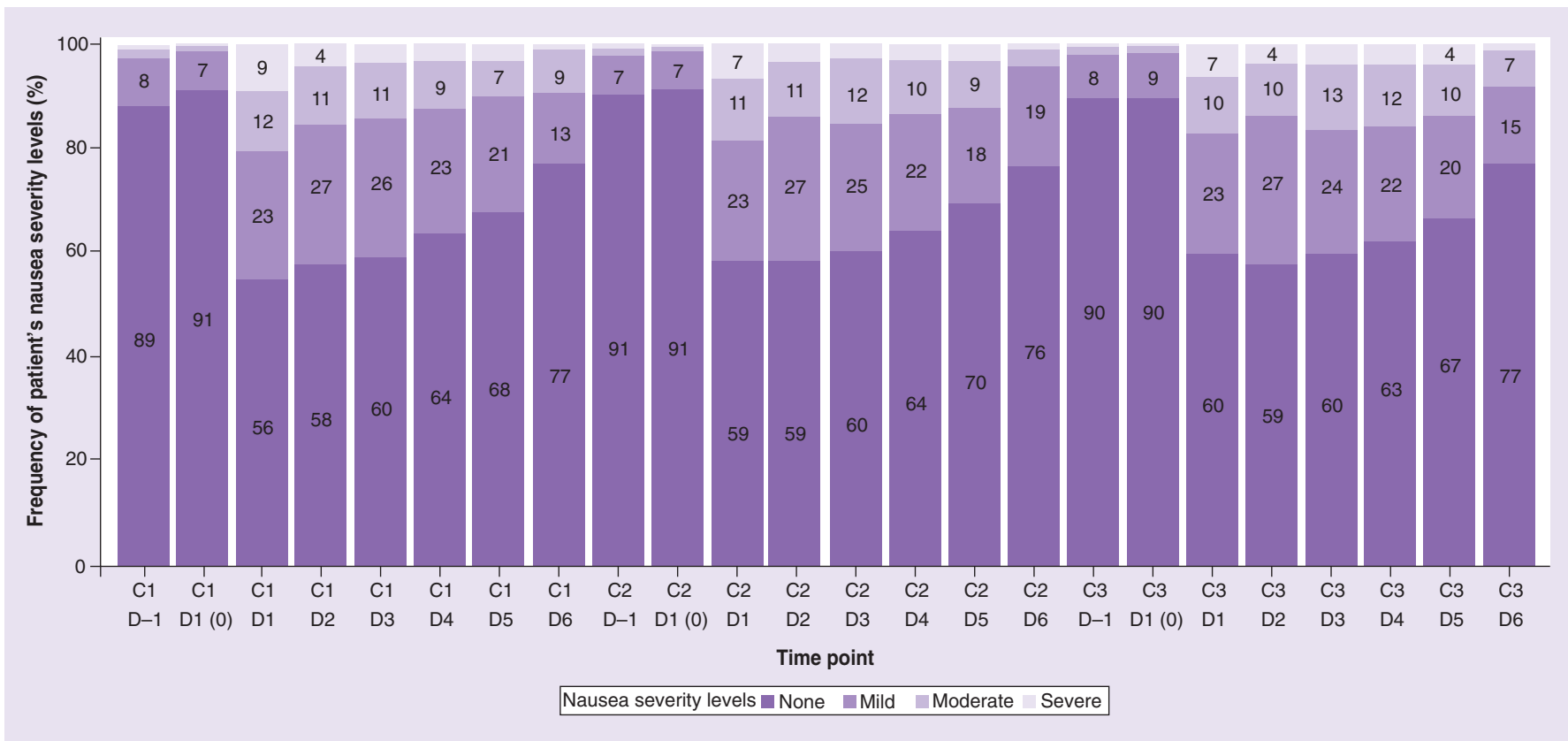

Figure 4. Nausea frequency and severity across days -1-6 of each chemotherapy cycle. D-1 indicates patients' assessment of nausea 24 $\mathrm{h}$ before chemotherapy; D1 (0) indicates nausea assessment immediately before chemotherapy; D1/2/3/4/5/6 indicate nausea assessment in the $24 / 48 / 72 / 96 / 120 / 144 \mathrm{~h}$, respectively, after the start of chemotherapy.

C: Cycle; D: Day.

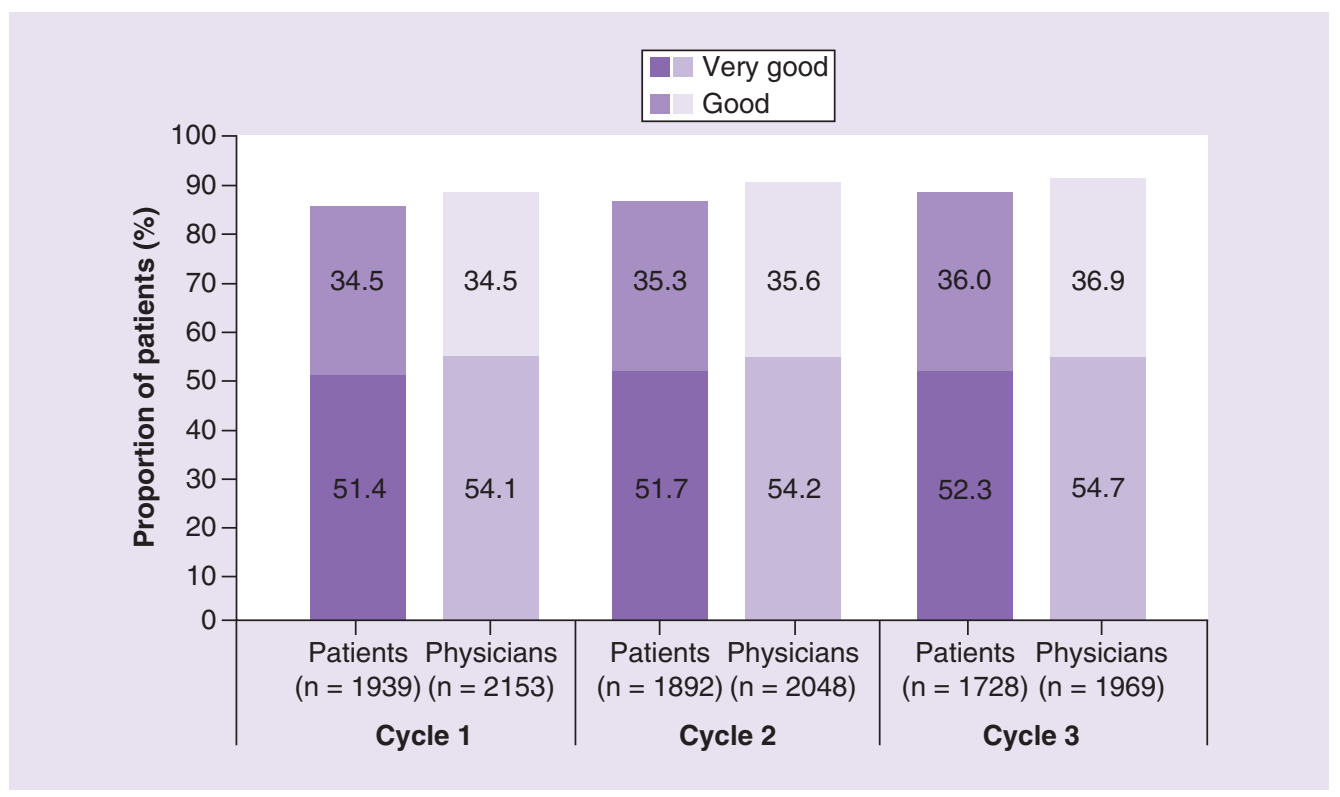

Figure 5. Perception of NEPA, netupitant-palonosetron, effectiveness by physicians and patients.

reported serious AEs, only $10(0.5 \%)$ patients had a total of 18 serious TRAEs and these corresponded to events that would be anticipated during treatment with chemotherapy; the most common serious TRAE was leukopenia (two events). The most common TRAEs, which occurred in $>1 \%$ of patients in the overall study period, were fatigue (3\%), constipation (3\%), nausea (2\%) and insomnia (2\%). There were no reports of NEPA-related deaths. 


\begin{tabular}{|c|c|c|c|c|}
\hline Number of patients with AEs (\%) & $\begin{array}{l}\text { Cycle } 1 \\
(n=2153)\end{array}$ & $\begin{array}{l}\text { Cycle } 2 \\
(n=2048)\end{array}$ & $\begin{array}{l}\text { Cycle } 3 \\
(n=1969)\end{array}$ & $\begin{array}{l}\text { Overall } \\
(n=2173)\end{array}$ \\
\hline Treatment-emergent AEs & $466(21.5)$ & $367(16.9)$ & $263(12.1)$ & $650(29.9)$ \\
\hline Treatment-related $\mathrm{AEs}^{\dagger}$ & $109(5.0)$ & $78(3.6)$ & $43(2.0)$ & $158(7.3)$ \\
\hline Serious AEs & $88(4.1)$ & $47(2.2)$ & $33(1.5)$ & $153(7.0)$ \\
\hline Serious treatment-related $\mathrm{AEs}^{\ddagger}$ & $6(0.3)$ & $4(0.2)$ & $1(<0.1)$ & $10(0.5)$ \\
\hline Treatment-related $\mathrm{AEs}^{\dagger}$ leading to death & 0 & 0 & 0 & 0 \\
\hline \multicolumn{5}{|l|}{ Treatment-related $\mathrm{AEs}^{\dagger}$ in $>1 \%$ of patients } \\
\hline - Fatigue & $43(2.0)$ & $14(0.6)$ & $11(0.5)$ & $64(3.0)$ \\
\hline - Constipation & $38(1.8)$ & $23(1.1)$ & $8(0.4)$ & $62(2.9)$ \\
\hline - Nausea & $19(0.9)$ & $20(0.9)$ & $14(0.6)$ & $38(1.8)$ \\
\hline - Insomnia & $25(1.2)$ & $9(0.4)$ & $1(<0.1)$ & 35 (1.6) \\
\hline \multicolumn{5}{|c|}{$\begin{array}{l}\dagger \text { Considered by the investigator as treatment related. } \\
\ddagger \text { A total of } 18 \text { serious treatment-related AEs were reported: leukopenia (two events), and increased ALT, increased AST, left bundle branch block, constipation, depression, } \\
\text { diarrhea, febrile neutropenia, increased GGT, hepatic failure, hyperbilirubinemia, hypertensive crisis, icterus, neutropenia, pancytopenia, serotonin syndrome and suicide } \\
\text { attempt (one event each). } \\
\text { AE: Adverse event. }\end{array}$} \\
\hline
\end{tabular}

\section{Discussion}

To our knowledge, this is the first prospective study that has evaluated the impact of NEPA usage in a realworld setting under routine clinical practice conditions. Of note, the proportion of patients completing the FLIE questionnaires was substantially higher compared with other trials on patient-reported outcomes. Pivotal clinical studies need to enroll patients with uniform basal characteristics and have strict requirements for accurate interpretation of the results in order to address regulatory needs; consequently, they do not reflect patient populations in the real world. Noninterventional studies offer the opportunity to evaluate drugs in a real-world setting, which means that patients with diverse baseline and disease characteristics from various clinical practice settings are included. Our current study included a total of 2173 patients with various tumor types enrolled at 162 centers throughout Germany. A substantial proportion of these patients had advanced disease at enrollment, with $25 \%$ of patients having metastatic tumors and $21 \%$ receiving palliative care. In cycle 1 , approximately $65 \%$ of patients received HEC (AC: 88\%; cisplatin: 12\%) and 36\% received MEC (carboplatin: 53\%; oxaliplatin: 21\%; other: $25 \%$; Table $1 \&$ Figure 1). Information regarding previous lines of chemotherapy was not systematically recorded in the electronic case report forms and was missing for most patients. However, given the fact that these patients were representative of a real-world cancer population and a number of them presented with an advanced stage of disease with metastases and/or were receiving palliative care, it would be expected that a considerable proportion of patients were non-naive to chemotherapy.

The primary objective of this study was to evaluate the effect of antiemetic prophylactic treatment with NEPA on the patients' quality of life, as measured using the FLIE questionnaire, and the impact of nausea and vomiting on daily-life activities. Importantly, $\geq 88 \%$ of patients completed the FLIE questionnaires at all three cycles. This completion rate is substantially higher compared with previous data from clinical trials on patient-reported outcomes and supports the validity of our results $[3,43,44]$. The fact that quality of life data were collected from a sustained number of patients across cycles (1886/1795/1698 patients in cycles $1 / 2 / 3$, respectively) suggests that there is no selection bias for patients with better quality of life. Overall, CINV was well-controlled as shown by the overall CR rate of approximately $83 \%$ across cycles. Vomiting was found to have little impact on quality of life in patients receiving NEPA prophylaxis, with the vast majority of patients (82-88\%) reporting NIDL due to vomiting during treatment with HEC and MEC for three cycles. The percentage of patients with NIDL due to nausea ranged from 54 to $66 \%$, and from 64 to $74 \%$ for the combined domain (Figure 2). There was a slight but consistent improvement in quality of life with regards to nausea, vomiting and combined domains across three cycles, with increases in NIDL in both the HEC and MEC settings being more pronounced for the nausea (HEC: 4\%; MEC: 7\%) and combined (HEC: 2\%; MEC: 7\%) domains. Overall, nausea and vomiting had a slightly greater impact on the quality of life in patients receiving HEC, compared with MEC (Figure 2). It is reassuring to note that the quality of life observed in the real-world cancer patient population in this study is in line with that previously reported in pivotal trials enrolling chemotherapy-naive patients receiving AC [28] or cisplatin [30] regimens (Supplementary Table 1) [28,30]. 
Antiemetic effectiveness was assessed as a secondary objective. The effectiveness of NEPA in terms of CR, emesis control and no need for rescue medication was high and, as was observed with the quality of life data, the results were in line with previous reports from clinical trials in the HEC and MEC settings (Figure 3, Table 2; Supplementary Table 2 [27-30]). In the overall period, the CR rate was approximately $83 \%$, the no emesis rate was $94 \%$, and the proportion of patients who did not need rescue medication was $87 \%$; these outcomes were maintained over three chemotherapy cycles. Effectiveness during the acute and delayed phases also resembled that observed in previous pivotal studies [27-30]. When considering effectiveness in terms of NSN, the rates in the acute/delayed/overall periods were slightly lower compared with previous results from Phase III trials, especially for the acute phase [2730]. Interestingly, both nausea frequency and severity were highest on the first day of chemotherapy treatment but gradually improved during the following days for each chemotherapy cycle (Figure 4).

Our results confirm that nausea is difficult to measure due to its subjective nature, and harder to control than vomiting, as has previously been reported [7], and emphasizes how clinically challenging this condition is. Nevertheless, NEPA-dexamethasone is the only $\mathrm{NK}_{1}$ RA-regimen that has shown a consistent benefit in nausea control, compared with other antiemetic prophylaxis using 5- $\mathrm{HT}_{3} \mathrm{RA}$-dexamethasone alone. In this large population of patients, our data show that the benefits of NEPA observed in clinical trials, with regard to CINV control, safety and quality of life, are maintained in the majority of patients in routine clinical practice in HEC and MEC settings (Figure $2 \&$ Table 2). The small differences in the results observed between previous randomized controlled trials and the present noninterventional study may be explained by differences in the characteristics of the patients. As mentioned above, our patient population is representative of a real-world population; hence, a minority of patients enrolled in this study were non-naive to chemotherapy and may have experienced CINV in previous lines of treatment. Moreover, approximately $10 \%$ of patients had experienced anticipatory nausea. Since occurrence of CINV in previous cycles and anticipatory nausea have both been identified as risk factors for CINV [22,45], patients who are chemotherapy non-naive and/or those with anticipatory nausea are usually excluded from randomized clinical trials. In addition, other patient-related factors, such as baseline ECOG performance status and the occurrence of nausea and vomiting associated with patients' comorbidities or concomitant medication, may also increase the risk for CINV in this study. Of note, a minority of patients received concomitant immunotherapeutic agents, all of low or minimal risk for CINV, per MASCC/ESMO guidelines; therefore, their contribution to the observed outcomes would be expected to be very limited.

A recent real-world, Phase IV study evaluated the effectiveness of the aprepitant/fosaprepitant-palonosetrondexamethasone regimen in the HEC setting [46]. Contrary to our results, lower levels of antiemetic efficacy were observed under real-world conditions, compared with results from randomized controlled trials. In the real-world setting, $49 \%$ of patients treated with HEC who were receiving an aprepitant/fosaprepitant-based prophylactic regimen had no CINV events [46], which contrasted with the results of a randomized Phase III trial, where $73 \%$ of patients assigned to receive an aprepitant-palonosetron-dexamethasone regimen had CR [47]. One possible explanation for such a result may be the complex administration schedule of the aprepitant-palonosetrondexamethasone regimen, which makes it difficult to implement in the real world.

Within our study, the level of antiemetic control achieved with NEPA was consistently ranked as 'very good' or 'good' by approximately $90 \%$ of physicians during the three chemotherapy cycles, and there was overall very good agreement between the effectiveness assessments by patients and physicians (Figure 5). In contrast, several other studies that have described patients' and physicians' perspectives on the incidence of CINV found there to be a disparity between the reported rates. This was attributed in part to the under-reporting of CINV occurrence by patients $[25,48,49]$ but also the overestimation of the effectiveness of antiemetic treatments by healthcare professionals both in the MEC $[19,50]$ and HEC [19,51] settings. The results of our large study that involved inpatients and outpatients from centers of all sizes found good concurrence between physicians' and patients' opinions of the effectiveness of NEPA, which may suggest that there is a clear benefit for patients from NEPA-based antiemetic prophylaxis.

In agreement with the results from the pivotal studies, NEPA was well-tolerated by patients in our study (Table 3). Under real-world conditions, where we report data from more than 2000 patients who presented with a wide range of comorbidities that often required concomitant medication, the safety profile of NEPA was in line with that reported in the pivotal trials [27-30] and consistent with that expected of $\mathrm{NK}_{1}$ RAs and 5-HT 3 RAs [1]. The most frequently reported TRAEs were low-grade fatigue and constipation. Of note, as per the study protocol, it was left to the investigator to determine whether there was a causal relationship between the observed AE and NEPA 
administration. A number of AEs that are typical of chemotherapy, and generally do not occur with NEPA, were categorized by the investigators as related to the administration of the study drug.

The current study has some limitations including the lack of a control arm and randomization, known intrinsic key limitations of real-world studies, needed for the external validity of the data. Additionally, while quality of life data are subjective by nature, validated FLIE questionnaires to collect those data daily from patients were used to minimize subjectivity. Finally, exhaustive patient data regarding prior chemotherapy, prior antiemetic prophylaxis and concomitant therapies and medications were not systematically documented and may limit further analyses and interpretation of results.

In summary, this noninterventional study has demonstrated that the NEPA-based prophylactic regimen for CINV is as effective and safe in the real world as previously observed in pivotal trials. In particular, the combination of NEPA plus dexamethasone can effectively prevent CINV in patients non-naive to chemotherapy who may also have additional CINV risk factors. The ease of administration of the NEPA regimen favors treatment compliance outside of the controlled conditions of clinical trials, and may prevent medication mistakes, particularly in those patients who need to take follow-up antiemetics at home. This contrasts with more complicated antiemetic schedules administered over multiple days, which could lead to lower levels of effectiveness in the real-world setting.

\section{Conclusion}

The real-world evidence regarding quality of life presented within supports the use of NEPA-based antiemetic prophylaxis in a heterogeneous population of cancer patients receiving HEC or MEC in Germany and confirms the effectiveness and safety of NEPA-based antiemetic prophylaxis under routine clinical practice settings.

\section{Summary points}

- Clinical trial data may be limited in their applicability, due to the strict inclusion and exclusion criteria.

- Real-world evidence enables deeper insights to be gained on effectiveness and adherence to treatment regimens in a variety of clinical settings.

- The ultimate goal of cancer supportive care is to improve patients' quality of life during treatment.

- Collection of patient-reported nausea and vomiting symptoms following antiemetic prophylaxis using netupitant-palonosetron (NEPA) revealed benefits in daily impact on quality of life in the domains of nausea, vomiting, and combined nausea and vomiting in patients treated with highly emetogenic chemotherapy and moderately emetogenic chemotherapy.

- NEPA was found to be effective and to promote high levels of complete response in the acute, delayed and overall phases, and was safe and well-tolerated.

- NEPA antiemetic effectiveness was rated highly both by patients and physicians.

- NEPA appears to provide similar quality of life benefits and to be as safe and effective in the real-world setting as was reported in the pivotal clinical trials.

- Real-world data suggest that the convenience of administration of NEPA may lead to high treatment compliance by patients during home administration.

Supplementary data

To view the supplementary data that accompany this paper please visit the journal website at: www.futuremedicine.com/doi/suppl/10.2217/fon-2020-0187

Acknowledgments

The authors thank the patients, investigators and the study teams.

Financial \& competing interests disclosure

The study described within this paper was sponsored by RIEMSER Pharma GmbH, Berlin, Germany. Helsinn Healthcare SA and RIEMSER Pharma GmbH also participated in the writing, review, and approval of the manuscript. M Karthaus is on the ad board and has received travel grants from Helsinn and RIEMSER. E Flahaut is a RIEMSER employee. The authors have no other relevant affiliations or financial involvement with any organization or entity with a financial interest in or financial conflict with the subject matter or materials discussed in the manuscript apart from those disclosed.

Editorial support and medical writing assistance were provided by Iratxe Abarrategui, PhD, CMPP, from Aptitude Health, The Hague, the Netherlands, funded by Helsinn Healthcare SA, Lugano, Switzerland. The authors are fully responsible for all content and editorial decisions for this manuscript. 
Clinical trial identification

DRKS00009316.

\section{References}

Papers of special note have been highlighted as: $\bullet$ of interest; $\bullet \bullet$ of considerable interest

1. Navari RM, Aapro M. Antiemetic prophylaxis for chemotherapy-induced nausea and vomiting. N. Engl. J. Med. 374(14), 1356-1367 (2016).

-• Comprehensive review of chemotherapy-induced nausea and vomiting.

2. Ballatori E, Roila F, Ruggeri B et al. The impact of chemotherapy-induced nausea and vomiting on health-related quality of life. Support. Care Cancer 15(2), 179-185 (2007).

3. Bloechl-Daum B, Deuson RR, Mavros P, Hansen M, Herrstedt J. Delayed nausea and vomiting continue to reduce patients' quality of life after highly and moderately emetogenic chemotherapy despite antiemetic treatment. J. Clin. Oncol. 24(27), 4472-4478 (2006).

4. Cohen L, de Moor CA, Eisenberg P, Ming EE, Hu H. Chemotherapy-induced nausea and vomiting: incidence and impact on patient quality of life at community oncology settings. Support. Care Cancer 15(5), 497-503 (2007).

5. Glaus A, Knipping C, Morant R et al. Chemotherapy-induced nausea and vomiting in routine practice: a European perspective. Support. Care Cancer 12(10), 708-715 (2004).

6. Basch E, Deal AM, Dueck AC et al. Overall survival results of a trial assessing patient-reported outcomes for symptom monitoring during routine cancer treatment. JAMA 318(2), 197-198 (2017).

7. Bošnjak SM, Gralla RJ, Schwartzberg L. Prevention of chemotherapy-induced nausea: the role of neurokinin-1 $\left(\mathrm{NK}_{1}\right)$ receptor antagonists. Support. Care Cancer 25(5), 1661-1671 (2017).

8. Aapro M, Molassiotis A, Dicato $\mathrm{M}$ et al. The effect of guideline-consistent antiemetic therapy on chemotherapy-induced nausea and vomiting (CINV): the Pan European Emesis Registry (PEER). Ann. Oncol. 23(8), 1986-1992 (2012).

9. Burmeister H, Aebi S, Studer C, Fey MF, Gautschi O. Adherence to ESMO clinical recommendations for prophylaxis of chemotherapy-induced nausea and vomiting. Support. Care Cancer 20(1), 141-147 (2012).

10. Gilmore JW, Peacock NW, Gu A et al. Antiemetic guideline consistency and incidence of chemotherapy-induced nausea and vomiting in US community oncology practice: INSPIRE study. J. Oncol. Pract. 10(1), 68-74 (2014).

11. Deutsche Krebsgesellschaft. [Guideline program oncology. Supportive therapy for oncological patients] (2019). www.leitlinienprogrammonkologie.de/fileadmin/user_upload/Downloads/Leitlinien/Supportivtherapie/LL_Supportiv_Langversion_1.2.pdf

12. Roila F, Molassiotis A, Herrstedt J et al. 2016 MASCC and ESMO guideline update for the prevention of chemotherapy- and radiotherapy-induced nausea and vomiting and of nausea and vomiting in advanced cancer patients. Ann. Oncol. 27(Suppl. 5), v119-v133 (2016).

13. Multinational Association of Supportive Care in Cancer. MASCC/ESMO Antiemetic Guidelines (2019). www.mascc.org/antiemetic-guidelines

- Most recent Multinational Association of Supportive Care in Cancer/European Society for Medical Oncology antiemetic guidelines.

14. Hesketh PJ, Kris MG, Basch E et al. Antiemetics: American Society of Clinical Oncology Clinical Practice Guideline update. J. Clin. Oncol. 35(28), 3240-3261 (2017).

- Most recent American Society of Clinical Oncology antiemetic guidelines.

15. National Comprehensive Cancer Network. NCCN Clinical Practice Guidelines in Oncology (NCCN Guidelines ${ }^{\circledR}$ ). Antiemesis. Version 1.2020 (2020). www.nccn.org/professionals/physician_gls/pdf/antiemesis.pdf

- Most recent National Comprehensive Cancer Network antiemetic guidelines.

16. Hesketh PJ, Kris MG, Grunberg SM et al. Proposal for classifying the acute emetogenicity of cancer chemotherapy. J. Clin. Oncol. 15(1), 103-109 (1997).

17. Grunberg SM, Warr D, Gralla RJ et al. Evaluation of new antiemetic agents and definition of antineoplastic agent emetogenicity-state of the art. Support. Care Cancer 19(Suppl. 1), S43-S47 (2011).

18. Jordan K, Chan A, Gralla RJ et al. 2016 updated MASCC/ESMO consensus recommendations: emetic risk classification and evaluation of the emetogenicity of antineoplastic agents. Support. Care Cancer 25(1), 271-275 (2017).

19. Aapro M, Ruffo P, Panteri R, Costa S, Piovesana V. Oncologist perspectives on chemotherapy-induced nausea and vomiting (CINV) management and outcomes: a quantitative market research-based survey. Cancer Rep. 1(4), e1127 (2018).

20. Aapro MS, Scotté F, Escobar Y et al. Evaluation of practice patterns for prevention of chemotherapy (CT)-induced nausea and vomiting (CINV) and antiemetic guidelines (GLs) adherence based on real-world prescribing data. Ann. Oncol. 29(Suppl.8), viii603, abstract 16810 (2018). 
21. Jordan K, Lux MP, Franceschetti A et al. Analysis of real-world prescribing patterns for the management of chemotherapy-induced nausea and vomiting and antiemetic guideline adherence. Presented at: Deutschen, Österreichischen und Schweizerischen Gesellschaften für Hämatologie und Medizinische Onkologie (DGHO) 2019 Congress. Berlin, Germany (2019).

22. Dranitsaris G, Molassiotis A, Clemons $\mathrm{M}$ et al. The development of a prediction tool to identify cancer patients at high risk for chemotherapy-induced nausea and vomiting. Ann. Oncol. 28(6), 1260-1266 (2017).

23. Clark-Snow RA, Affronti ML, Rittenberg CN. Chemotherapy-induced nausea and vomiting (CINV) and adherence to antiemetic guidelines: results of a survey of oncology nurses. Support. Care Cancer 26(2), 557-564 (2018).

24. Dielenseger P, Börjeson S, Vidall C, Young A, Jahn P. Evaluation of antiemetic practices for prevention of chemotherapy-induced nausea and vomiting (CINV): results of a European oncology nurse survey. Support. Care Cancer 27(11), 4099-4106 (2019).

25. Vidall C, Fernández-Ortega P, Cortinovis D, Jahn P, Amiani B, Scotté F. Impact and management of chemotherapy/radiotherapy-induced nausea and vomiting and the perceptual gap between oncologists/oncology nurses and patients: a cross-sectional multinational survey. Support. Care Cancer 23(11), 3297-3305 (2015).

26. Rojas $\mathrm{C}$, Slusher BS. Pharmacological mechanisms of 5- $\mathrm{HT}_{3}$ and tachykinin $\mathrm{NK}_{1}$ receptor antagonism to prevent chemotherapy-induced nausea and vomiting. Eur. J. Pharmacol. 684(1-3), 1-7 (2012).

27. Hesketh PJ, Rossi G, Rizzi G et al. Efficacy and safety of NEPA, an oral combination of netupitant and palonosetron, for prevention of chemotherapy-induced nausea and vomiting following highly emetogenic chemotherapy: a randomized dose-ranging pivotal study. Ann. Oncol. 25(7), 1340-1346 (2014).

- Pivotal study to determine the dose of netupitant to combine with palonosetron $0.50 \mathrm{mg}$ for further clinical evaluation of netupitant-palonosetron (NEPA).

28. Aapro M, Rugo H, Rossi G et al. A randomized Phase III study evaluating the efficacy and safety of NEPA, a fixed-dose combination of netupitant and palonosetron, for prevention of chemotherapy-induced nausea and vomiting following moderately emetogenic chemotherapy. Ann. Oncol. 25(7), 1328-1333 (2014).

- Pivotal study that evaluates the efficacy of NEPA in patients receiving anthracycline-cyclophosphamide.

29. Gralla RJ, Bosnjak SM, Hontsa A et al. A Phase III study evaluating the safety and efficacy of NEPA, a fixed-dose combination of netupitant and palonosetron, for prevention of chemotherapy-induced nausea and vomiting over repeated cycles of chemotherapy. Ann. Oncol. 25(7), 1333-1339 (2014).

- Pivotal study that evaluates the safety and efficacy of NEPA over multiple cycles of highly and moderately emetogenic chemotherapy.

30. Zhang L, Lu S, Feng J et al. A randomized Phase III study evaluating the efficacy of single-dose NEPA, a fixed antiemetic combination of netupitant and palonosetron, versus an aprepitant regimen for prevention of chemotherapy-induced nausea and vomiting (CINV) in patients receiving highly emetogenic chemotherapy (HEC). Ann. Oncol. 29(2), 452-458 (2018).

- Phase III study comparing the antiemetic efficacy of NEPA and aprepitant regimens.

31. Hesketh PJ, Grunberg SM, Gralla RJ et al. The oral neurokinin-1 antagonist aprepitant for the prevention of chemotherapy-induced nausea and vomiting: a multinational, randomized, double-blind, placebo-controlled trial in patients receiving high-dose cisplatin - the Aprepitant Protocol 052 Study Group. J. Clin. Oncol. 21(22), 4112-4119 (2003).

32. Warr DG, Hesketh PJ, Gralla RJ et al. Efficacy and tolerability of aprepitant for the prevention of chemotherapy-induced nausea and vomiting in patients with breast cancer after moderately emetogenic chemotherapy. J. Clin. Oncol. 23(12), 2822-2830 (2005).

33. Rapoport BL, Chasen MR, Gridelli C et al. Safety and efficacy of rolapitant for prevention of chemotherapy-induced nausea and vomiting after administration of cisplatin-based highly emetogenic chemotherapy in patients with cancer: two randomised, active-controlled, double-blind, Phase III trials. Lancet Oncol. 16(9), 1079-1089 (2015).

34. Schwartzberg LS, Modiano MR, Rapoport BL et al. Safety and efficacy of rolapitant for prevention of chemotherapy-induced nausea and vomiting after administration of moderately emetogenic chemotherapy or anthracycline and cyclophosphamide regimens in patients with cancer: a randomised, active-controlled, double-blind, Phase III trial. Lancet Oncol. 16(9), 1071-1078 (2015).

35. Aapro M, Karthaus M, Schwartzberg L et al. NEPA, a fixed oral combination of netupitant and palonosetron, improves control of chemotherapy-induced nausea and vomiting (CINV) over multiple cycles of chemotherapy: results of a randomized, double-blind, Phase III trial versus oral palonosetron. Support. Care Cancer 25(4), 1127-1135 (2017).

36. Schwartzberg L, Roeland E, Andric Z et al. Phase III safety study of intravenous NEPA: a novel fixed antiemetic combination of fosnetupitant and palonosetron in patients receiving highly emetogenic chemotherapy. Ann. Oncol. 29(7), 1535-1540 (2018).

37. Schwartzberg L, Navari R, Clark-Snow R et al. Phase IIIb safety and efficacy of intravenous NEPA for prevention of chemotherapy-induced nausea and vomiting (CINV) in patients with breast cancer receiving initial and repeat cycles of anthracycline/cyclophosphamide (AC) chemotherapy. Oncologist 25, e589-e597 (2020).

38. Akynzeo ${ }^{\circledR}$ (netupitant and palonosetron) capsules; Akynzeo ${ }^{\circledR}$ (fosnetupitant and palonosetron) for injection, prescribing information. Dublin, Ireland (2018). Helsinn Birex Pharmaceuticals Ltd, Dublin, Ireland

39. EMA. Summary of opinion (post authorization). Akynzeo (fosnetupitant/palonosetron). EMA/CHMP/670824/2019. www.ema.europa.eu/en/documents/smop-initial/chmp-summary-positive-opinion-akynzeo_en.pdf 
40. Jordan K, Aapro M, Kaasa S et al. European Society for Medical Oncology (ESMO) position paper on supportive and palliative care. Ann. Oncol. 29(1), 36-43 (2018).

41. Basch E, Deal AM, Kris MG et al. Symptom monitoring with patient-reported outcomes during routine cancer treatment: a randomized controlled trial. J. Clin. Oncol. 34(6), 557-565 (2016).

42. Martin AR, Pearson JD, Cai B, Elmer M, Horgan K, Lindley C. Assessing the impact of chemotherapy-induced nausea and vomiting on patients' daily lives: a modified version of the Functional Living Index-Emesis (FLIE) with 5-day recall. Support. Care Cancer 11(8), 522-527 (2003).

43. Ganz PA, Gotay CC. Use of patient-reported outcomes in Phase III cancer treatment trials: lessons learned and future directions. J. Clin. Oncol. 25(32), 5063-5069 (2007).

44. Bell ML, Fairclough DL. Practical and statistical issues in missing data for longitudinal patient-reported outcomes. Stat. Methods Med. Res. 23(5), 440-459 (2014).

45. Molassiotis A, Lee PH, Burke TA et al. Anticipatory nausea, risk factors, and its impact on chemotherapy-induced nausea and vomiting: results from the Pan European Emesis Registry study. J. Pain Symptom Manage. 51(6), 987-993 (2016).

46. Schwartzberg LS, McLaughlin T, Geller RB, Gabrail NY, Marks SM. Real-world efficacy: intravenous palonosetron three-drug regimen for chemotherapy-induced nausea and vomiting with highly emetogenic chemotherapy. J. Comp. Eff. Res. 7(12), 1161-1170 (2018).

47. Navari RM, Gray SE, Kerr AC. Olanzapine versus aprepitant for the prevention of chemotherapy-induced nausea and vomiting: a randomized Phase III trial. J. Support. Oncol. 9(5), 188-195 (2011).

48. Di Maio M, Gallo C, Leighl NB et al. Symptomatic toxicities experienced during anticancer treatment: agreement between patient and physician reporting in three randomized trials. J. Clin. Oncol. 33(8), 910-915 (2015).

49. Childs DS, Looker S, Le-Rademacher J, Ridgeway JL, Breitkopf CR, Jatoi A. What occurs in the other $20 \%$ of cancer patients with chemotherapy-induced nausea and vomiting (CINV)? A single-institution qualitative study. Support. Care Cancer 27(1), 249-255 (2019).

50. Escobar Y, Cajaraville G, Virizuela JA et al. Incidence of chemotherapy-induced nausea and vomiting with moderately emetogenic chemotherapy: ADVICE (Actual Data of Vomiting Incidence by Chemotherapy Evaluation) study. Support. Care Cancer 23(9), 2833-2840 (2015).

51. Majem M, Moreno ME, Calvo N et al. Perception of healthcare providers versus patient reported incidence of chemotherapy-induced nausea and vomiting after the addition of NK-1 receptor antagonists. Support. Care Cancer 19(12), 1983-1990 (2011). 
